\title{
ORIGINAL
}

\section{Veno-venous extracorporeal membrane oxygenation (vv-ECMO) for severe respiratory failure in adult cancer patients: a retrospective multicenter analysis}

Matthias Kochanek ${ }^{*} \mathbb{0}$, Jan Kochanek ${ }^{1}$ Boris Böll ${ }^{1}$, Dennis A. Eichenauer ${ }^{1}$, Gernot Beutel ${ }^{2}$, Hendrik Bracht ${ }^{3}$, Stephan Braune ${ }^{4}$, Florian Eisner ${ }^{5}$, Sigrun Friesecke ${ }^{6}$, Ulf Günther ${ }^{7}$, Gottfried Heinz ${ }^{8}$, Michael Hallek? , Christian Karagiannidis ${ }^{9}$, Stefan Kluge ${ }^{4}$, Klaus Kogelmann ${ }^{10}$, Pia Lebiedz ${ }^{11}$, Philipp M. Lepper ${ }^{12}$, Tobias Liebregts ${ }^{13}$, Catherina Lueck ${ }^{2}$, Ralf M. Muellenbach ${ }^{14}$, Matthias Hansen ${ }^{15}$, Christian Putensen ${ }^{16}$, Peter Schellongowski ${ }^{17}$, Jens-Christian Schewe ${ }^{16}$, Kathrin Schumann-Stoiber ${ }^{18}$, Frederik Seiler ${ }^{12}$, Peter Spieth ${ }^{19}$, Steffen Weber-Carstens ${ }^{20}$, Daniel Brodie ${ }^{21,22}$, Elie Azoulay ${ }^{23}$ and Alexander Shimabukuro-Vornhagen ${ }^{1}$

() 2022 The Author(s)

\begin{abstract}
Purpose: The question of whether cancer patients with severe respiratory failure benefit from veno-venous extracorporeal membrane oxygenation ( $v \mathrm{v}$-ECMO) remains unanswered. We, therefore, analyzed clinical characteristics and outcomes of a large cohort of cancer patients treated with vv-ECMO with the aim to identify prognostic factors.
\end{abstract}

Methods: 297 cancer patients from 19 German and Austrian hospitals who underwent vv-ECMO between 2009 and 2019 were retrospectively analyzed. A multivariable cox proportional hazards analysis for overall survival was performed. In addition, a propensity score-matched analysis and a latent class analysis were conducted.

Results: Patients had a median age of 56 (IQR 44-65) years and 214 (72\%) were males. 159 (54\%) had a solid tumor and 138 (47\%) a hematologic malignancy. The 60-day overall survival rate was $26.8 \%$ (95\% Cl 22.1-32.4\%). Low platelet count (HR 0.997, 95\% Cl 0.996-0.999; $p=0.0001$ per 1000 platelets/ $\mu$ ), elevated lactate levels (HR 1.048, 95\% Cl 1.012-1.084; $p=0.0077$ ), and disease status (progressive disease [HR 1.871,95\% Cl 1.081-3.238; $p=0.0253]$, newly diagnosed [HR 1.571, 95\% Cl 1.044-2.364; $p=0.0304]$ ) were independent adverse prognostic factors for overall survival. A propensity score-matched analysis with patients who did not receive ECMO treatment showed no significant survival advantage for treatment with ECMO.

Conclusion: The overall survival of cancer patients who require vv-ECMO is poor. This study shows that the value of VV-ECMO in cancer patients with respiratory failure is still unclear and further research is needed. The risk factors identified in the present analysis may help to better select patients who may benefit from vv-ECMO.

Keywords: Extracorporeal membrane oxygenation, Extracorporeal circulation, ECMO, Cancer, Respiratory failure, Acute respiratory distress syndrome, ARDS

\footnotetext{
*Correspondence: Matthias.Kochanek@uk-koeln.de

${ }^{1}$ First Department of Internal Medicine, Faculty of Medicine

and University Hospital Cologne, Center of Integrated Oncology Aachen

Bonn Cologne Dusseldorf, University of Cologne, University Hospital

Cologne, Kerpener Str. 62, 50937 Cologne, Germany

Full author information is available at the end of the article
}

\section{照




\section{Introduction}

Cancer patients are at increased risk for the development of respiratory failure, either due to the underlying malignancy or due to cancer treatment-related complications [1]. Respiratory failure represents the most common cause for intensive care unit (ICU) admission among patients with a malignancy [2-5]. Despite substantial improvements made in recent years, mortality attributable to acute respiratory failure remains high in this patient population [6].

Acute respiratory failure and acute respiratory distress syndrome (ARDS) are associated with a mortality rate between 28 and 50\% [7, 8]. Despite improvements in recent years, mortality in patients with cancer remains to be worse [9]. Veno-venous extracorporeal membrane oxygenation (vv-ECMO) is a treatment option for patients with severe ARDS in whom conventional mechanical ventilation fails [10-12]. Vv-ECMO can serve as an artificial lung, providing extracorporeal gas exchange to maintain adequate oxygenation and carbon dioxide removal with the aim to minimize ventilatorinduced lung injury until the cause of severe respiratory failure has resolved. Over the last decade, technological advances of ECMO circuits and improvements in the clinical management have resulted in better overall outcomes in patients undergoing vv-ECMO [13-15]. Recent data from the registry of the Extracorporeal Life Support Organization (ELSO) indicated a hospital discharge rate of $58 \%$ among non-cancer patients with severe ARDS receiving vv-ECMO [16].

Despite these improvements, the selection of patients eligible for vv-ECMO is an ongoing controversy. This is especially true for cancer patients given the prognostic uncertainty with regard to the underlying malignancy, the increased rate of infections relative to the general ICU population, and a high risk of severe vv-ECMOrelated adverse events [17-21]. Consequently, the Guideline from the Extracorporeal Life Support Organization (ELSO) includes immunosuppression as relative contraindication for the application of vv-ECMO [22]. Data on the use of vv-ECMO in cancer patients are limited. We, therefore, conducted a large multicenter retrospective study to describe clinical characteristics, outcomes and prognostic factors of cancer patients treated with vv-ECMO.

\section{Patients and methods}

\section{Patients}

Cancer patients $\geq 18$ years, with acute hypoxemic respiratory failure, who underwent vv-ECMO in 17 German and 2 Austrian hospitals between 1 January, 2009 through 31 December, 2019 were included in this

\section{Take-home message}

The overall survival of cancer patients who require $\mathrm{v} v-\mathrm{ECMO}$ is poor and, therefore, vv-ECMO should only be offered to selected patients. We identified disease status, low platelet count and high lactate levels are indicators of poor prognosis that should be accounted for in the decision to provide $\mathrm{v} v-\mathrm{ECMO}$.

retrospective multicenter study. Patients were eligible if they had been diagnosed with cancer within 5 years prior to the start of vv-ECMO treatment or if cancer was newly diagnosed with full confirmation during vv-ECMO treatment. All patients in this latter category were diagnosed with a malignant disease prior to initiation of ECMO either by imaging and/or initial histologic workup tumor tissue samples but were either missing final confirmation of their precise tumor diagnosis and/or had not initiated treatment yet. In none of the included patients was the cancer diagnosis made after ECMO initiation as a chance finding.

The study was conducted in accordance with the amended Declaration of Helsinki 2013. The study protocol was approved by the Institutional Review Board of the University of Cologne. Given the retrospective nature and the non-interventional design of the study, informed consent was waived.

\section{Data}

Age, gender, type and stage of the malignancy, date of diagnosis, type and date of cancer therapies (i.e., chemo-, immuno-, or radiotherapy, autologous or allogeneic stem cell transplantation) as well as the remission status were recorded (for details see Supplementary Information methods). A modified Charlson Comorbidity Index (mCCI) (excluding age and cancer diagnosis) was assessed to account for concurrent or preexisting conditions. Arterial blood gas parameters $\left(\mathrm{pH}, \mathrm{paO}_{2}\right.$ and $\mathrm{paCO}_{2}$, lactate), $\mathrm{P} / \mathrm{F}$ ratio and laboratory tests including white blood cell count, platelet count, and hemoglobin, were also recorded. In addition, the following variables were assessed: vasopressor use, transfused blood products (platelets and packed red blood cells (PRBC)), duration of vv-ECMO, duration of mechanical ventilation, length of ICU and hospital stay, other procedure-related complications (trauma or bleeding related to cannula insertion, clotting events), severe bleeding complications (according to the International Society of Thrombosis and Haemostasis (ISTH) (at least two units of PRBC, surgical interventions or intracerebral hemorrhage [23]), ICU and hospital survival, time of death, if applicable, as well as time of the last visit.

The decision to withdraw/withhold ECMO support or other life-sustaining therapies was made in accordance 
with the German Society of Hematology and Medical Oncology (DGHO) and Austrian Society of Hematology and Oncology (OeGHO) consensus guidelines for cancer patients requiring intensive care support [5].

In addition to the data from the primary cohort, we used data from the EFRAIM study, a multinational prospective cohort study, which enrolled 1611 immunocompromised patients with acute hypoxemic respiratory failure [24]. Inclusion criteria were $\geq 18$ years; acute hypoxemic respiratory failure; need for more than $6 \mathrm{~L} /$ min oxygen; respiratory symptom duration less than $72 \mathrm{~h}$ and non-AIDS-related immune deficiency defined as hematologic malignancy or solid tumor (active or in remission for less than 5 years, including recipients of autologous or allogeneic stem cell transplantation). This study evaluated the effects of the initial management strategy on patient outcome, i.e., endotracheal intubation rate and mortality. $51.9 \%$ and $35.2 \%$ of the patients enrolled in the study had a hematologic malignancy or a solid tumor, respectively.

\section{Statistical analysis}

Baseline categorical variables were reported as counts and percentages and were compared using the chi-square test. Baseline continuous variables were reported as medians and interquartile ranges (IQR) and were compared with the Mann-Whitney $U$-test. Overall survival was described with Kaplan-Meier survival curves, and the comparisons between groups were performed using the log-rank test. Model-based multiple imputation was used to account for missing values of variables. Missing data were imputed using a random forest algorithm.

Multivariable hierarchical models with random intercept per center were used to identify factors independently associated with survival. The results are reported using adjusted odds ratios (OR) for clinical covariates with 95\%-confidence intervals (CI). The multivariable mixed-effect Cox proportional hazards models and generalized linear mixed-effect models were computed in $\mathrm{R}$ using the coxme and lme4 package, respectively. Sensitivity analysis was conducted and confirmed robustness of the results.

To compare the outcome of our cohort with patients managed without the use of ECMO, a propensity scorematched analysis was performed (see Supplementary Information: Propensity score matching). Latent class analysis (LCA) was used to derive latent subgroups within our patient cohort (see Supplementary Information: Latent class analysis).

All $p$ values are two sided. $P$ values $<0.05$ were considered statistically significant, without correction for multiple testing. Analyses were performed with R software, version 4.0.3 ( $\mathrm{R}$ Foundation).

\section{Results}

A total of 297 patients were included in the study. Baseline characteristics are summarized in Table 1 . There were significant clinical differences between patients with hematologic malignancies and solid tumors (Table 1). Median baseline $\mathrm{PaO}_{2} / \mathrm{FiO}_{2}$ ratio was $71 \mathrm{mmHg}$ [IQR 54-98 mmHg]. The $\mathrm{PaO}_{2} / \mathrm{FiO}_{2}$ ratio was lower in the group of patients with hematologic malignancies $(67 \mathrm{mmHg}$ [54-89 $\mathrm{mmHg}$ ] versus $73 \mathrm{mmHg}$ [59-104 mmHg]; $\mathrm{p}=0.015)$. The median time from endotracheal intubation to vv-ECMO initiation was 2 days (IQR 0.65-8.62 days). Before vv-ECMO vasopressor support was necessary in $92.6 \%$ of patients and $16.2 \%$ required renal replacement therapy; 42 (14.1\%) patients had undergone cardiopulmonary resuscitation (CPR) within 10 days before vv-ECMO was initiated.

The median follow-up was 182 days (95\% CI 115391 days). The 60-day overall survival for the entire study population was $26.8 \%$ (95\% CI $22.1-32.4 \%$ ). Baseline clinical characteristics that were associated with worse survival in multivariable analysis included disease status (newly diagnosed: HR 1.571 (95\% CI 1.0442.364; $p=0.0304)$; progressive disease: HR 1.871 (95\% CI 1.081-3.238; $p=0.0253)$ ), lower platelet counts (HR 0.997 (95\% CI 0.996-0.999; $p=0.0001$ per 1000 platelets/ $\mu \mathrm{l})$ and elevated serum lactate (HR 1.048 (95\% CI 1.012-1.084; $p=0.0077$ ) (Fig. 1, Supplementary Information Fig. 1).

An additional prognostic factor in patients with hematologic malignancies was serum lactate at baseline, a well-established prognostic factor in critically ill patients $[35,36]$. In patients with solid tumors, only serum creatinine had independent prognostic impact in addition to platelet count. This finding in cancer patients with solid tumors is in line with many other studies on a variety of critically ill patients showing that decreased renal function is associated with worse outcome [37, 38].

Patients with hematologic malignancies had a lower median overall survival than patients with solid tumors (Supplementary Information Fig. 2). 60-day overall survival was $23 \%(16.8-31.5 \% 95 \% \mathrm{CI})$ for patients with hematologic malignancies and 30\% (23.6-38.1\% 95\% CI) for patients with solid tumors $(p=0.048)$. In multivariable analysis, only platelet count was associated with a decreased overall survival in both patients with hematologic malignancies $(0.997$ (95\% CI 0.994-0.999; $p=0.0372)$ ) and solid tumors 0.998 (95\% CI $0.996-$ 0.999; $p=0.0067$ ) (Supplementary Information Figs. 3 and 4). In patients with hematologic malignancies, progressive disease (HR 3.642 (95\% CI 1.693-7.838; $p=0.0009)$ and higher lactate levels (HR 1.093 (95\% CI $1.040-1.149 ; p=0.0004)$ were independent adverse prognostic factors. In addition to the platelet count, 
Table 1 Baseline Characteristics and comparison between patients with hematologic malignancies and solid tumors

\begin{tabular}{|c|c|c|c|c|}
\hline & Overall & Hematologic malignancies & Solid tumors & $p$-value \\
\hline No. of patients (\%) & 297 & $138(46.4)$ & $159(53.5)$ & \\
\hline Age (median [IQR]) & $56[44-65]$ & $48[35.2-58.7]$ & $59[52.5-67]$ & $<0.001$ \\
\hline Sex, male (\%) & $214(72.1)$ & $99(71.7)$ & $115(72.3)$ & \\
\hline \multicolumn{5}{|l|}{ Leading cause for ICU admission } \\
\hline Respiratory failure & $203(68.4)$ & $110(79.7)$ & $93(58.5)$ & $<0.001$ \\
\hline Surgery & $41(13.8)$ & $2(1.4)$ & $39(24.5)$ & \\
\hline Non-pulmonary infection & $25(8.4)$ & $12(8.7)$ & $13(8.2)$ & \\
\hline Cardiac event & $2(0.7)$ & $0(0)$ & $2(1.3)$ & \\
\hline Other & $26(8.8)$ & $14(10.1)$ & $12(7.5)$ & \\
\hline \multicolumn{5}{|l|}{ Reason for vv-ECMO (\%) } \\
\hline Respiratory failure & $284(95.6)$ & $138(100)$ & $146(91.9)$ & 0.003 \\
\hline ECMO-facilitated surgery & $13(4.4)$ & $0(0)$ & $13(8.1)$ & \\
\hline RESP score (median [IQR]) & $-1[-3,2]$ & $0[-2-2]$ & $-1[-4-1]$ & $<0.001$ \\
\hline $\mathrm{P} / \mathrm{F}$ ratio (median $[\mathrm{IQR}]$ ) & $70.5[54.5,98.26]$ & $67.05[53.78-89.37]$ & $73.33[58.6-103.97]$ & 0.017 \\
\hline $\begin{array}{l}\text { Time between intubation and vv-ECMO (in days) (median } \\
{[\text { [QR]) }}\end{array}$ & $2[0,8]$ & $1.5[0,7]$ & $3[0.5,9]$ & 0.051 \\
\hline Noradrenalin equivalents (median [IQR]) & $0.33[0.1,0.9]$ & $0.34[0.12-0.96]$ & $0.3[0.1-0.86]$ & 0.583 \\
\hline Vasopressors before vv-ECMO & $275(92.6)$ & $129(93.5)$ & $146(91.8)$ & 0.748 \\
\hline Renal replacement therapy before vv-ECMO & $48(16.2)$ & $25(18.1)$ & $23(14.5)$ & 0.487 \\
\hline Mechanical ventilation last 180d & $60(20.2)$ & $8(5.8)$ & $52(32.7)$ & $<0.001$ \\
\hline Cardiopulmonary resuscitation before vv-ECMO & $42(14.1)$ & $15(10.9)$ & $27(17)$ & 0.180 \\
\hline mCCI total (median [IQR]) & $0[0-1]$ & $0[0-1]$ & $1[0-2]$ & 0.003 \\
\hline \multicolumn{5}{|l|}{ Disease status at admission to ICU (\%) } \\
\hline CR & $133(44.8)$ & $58(42)$ & $75(47.2)$ & 0.697 \\
\hline Newly diagnosed & $56(18.9)$ & $30(21.7)$ & $26(16.4)$ & \\
\hline PR/Controlled & $70(23.6)$ & $33(23.9)$ & $37(23.3)$ & \\
\hline Progression & $22(7.4)$ & $11(8)$ & $11(6.9)$ & \\
\hline Unknown & $16(5.4)$ & $6(4.3)$ & $10(6.3)$ & \\
\hline $\begin{array}{l}\text { Cancer treatment (chemotherapy, radiotherapy, immuno- } \\
\text { therapy) within } 90 \text { days before } \mathrm{v} v \text {-ECMO initiation }\end{array}$ & $144(48.5)$ & 85 (61.6) & $59(37.1)$ & $<0.001$ \\
\hline Previous autologous HSCT & $13(4.4)$ & $13(9.4)$ & $0(0)$ & $<0.001$ \\
\hline Previous allogeneic HSCT & $50(16.8)$ & $50(36.2)$ & $0(0)$ & $<0.001$ \\
\hline GVHD on admission to ICU & $23(7.7)$ & $23(16.7)$ & $0(0)$ & $<0.001$ \\
\hline Leukocytes G/L (median [IQR]) & $12.39[5,18.2]$ & $6.08[1.81-13.46]$ & $14.86[11.25-20.1]$ & $<0.001$ \\
\hline Neutropenia (<500/microL) & $42(14.1)$ & $38(27.5)$ & $4(2.5)$ & $<0.001$ \\
\hline Hemoglobin g/dl (median [IQR]) & $9.28[8.3-10.2]$ & $9.1[8.3-10.2]$ & $9.34[8.4-10.08]$ & 0.323 \\
\hline Platelets G/L (median [IQR]) & $135[44-242]$ & $48[21.25-135]$ & $200[126.53-313]$ & $<0.001$ \\
\hline Creatinine mg/dl (median [IQR]) & $1.15[0.8-1.7]$ & $1.23[0.75-1.91]$ & $1.12[0.86-1.63]$ & 0.847 \\
\hline Lactate $(\mathrm{mmol} / \mathrm{l})$ & $2.07[1.33-4.4]$ & $2[1.33-4.77]$ & $2.1[1.33-3.9]$ & 0.411 \\
\hline
\end{tabular}

Data are presented as $\mathrm{n}(\%)$ for categorical variables and median (IQR) for continuous variables

ICU intensive care unit, $\mathrm{mCCl}$ modified Charlson Comorbidity Index (CCI) (excluded age and cancer diagnosis), HSCT hematopoietic stem cell transplantation, RESP Respiratory ECMO Survival Prediction Score, $C R$ complete remission, $P R$ partial remission, GVHD Graft versus host disease

higher serum creatinine was independently associated with a lower overall survival in patients with solid tumors (HR 1.332 (95\% CI 1.031-1.721; $p=0.0284$ ).

The need of transfusion of blood products was frequent in both patient groups. $94.6 \%$ and $63 \%$ of the patients required at least one unit of PRBCs or platelet concentrate, respectively (Table 2). More than half of the patients $(52.5 \%)$ received $\geq 10$ PRBC units (hematologic malignancies: $52.2 \%$; solid tumors: $52.8 \% ; p=0.974)$. Given the significantly lower platelet count at baseline, patients with hematologic malignancies more often received $\geq 10$ platelet transfusions (hematologic malignancies: $39.9 \%$; solid tumors: $9.4 \%$; 


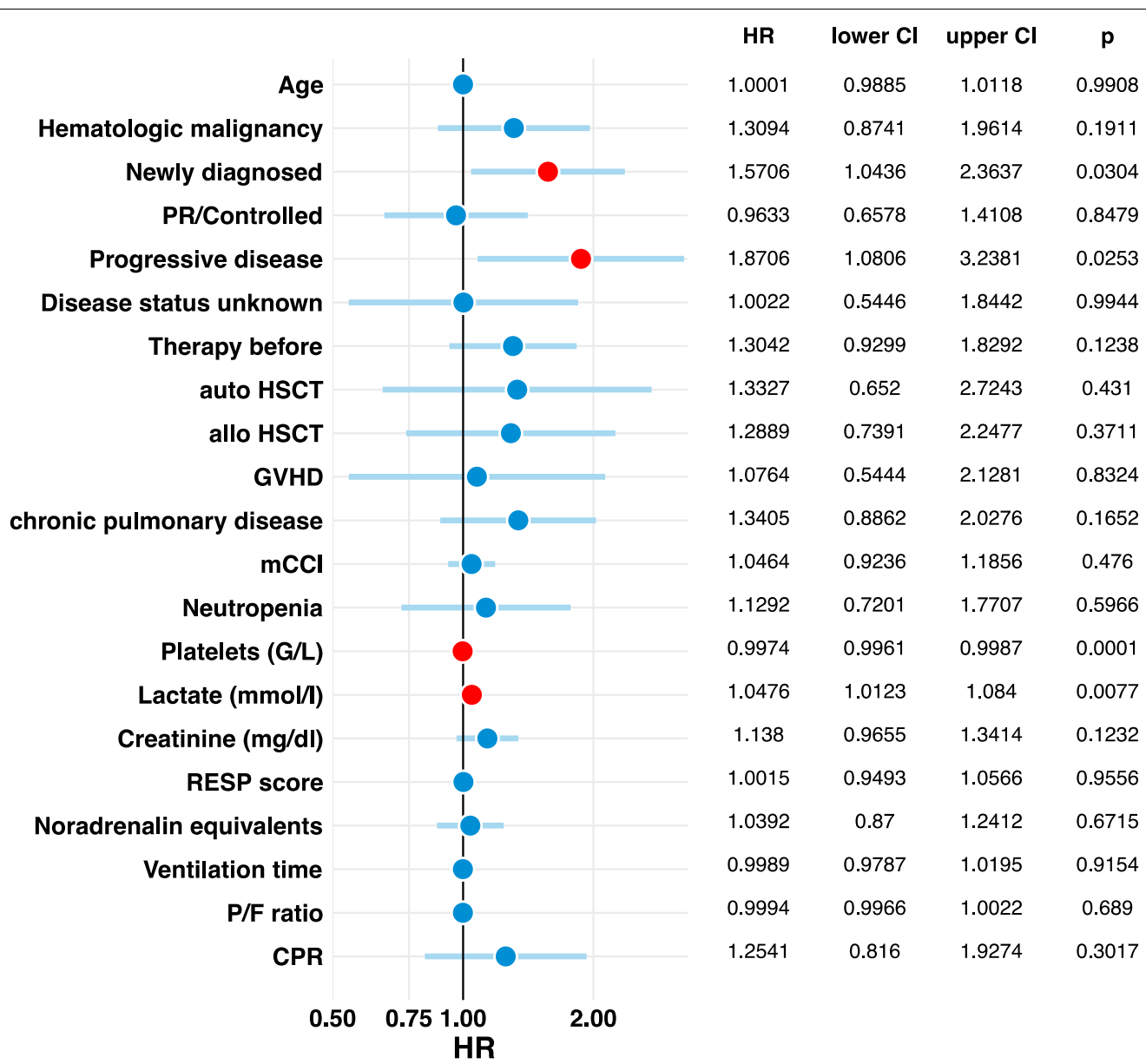

Fig. 1 Forest plots summarizing the results of the multivariate analysis for all patients. Shown are the adjusted hazard ratios for overall survival and 95\% confidence intervals. (Abbreviations: $\mathrm{mCCl}$ modified Charlson Comorbidity Index (CCI) (excluded age and cancer diagnosis); GVHD Graft versus host disease; HSCT hematopoietic stem cell transplantation; RESP Score Respiratory ECMO Survival Prediction Score; CPR Cardiopulmonary Resuscitation)

$p<0.001)$. ECMO-related severe bleeding occurred in $38 \%$ of patients (hematologic malignancies: $44.2 \%$; solid tumors: $32.7 \% ; p=0.055)$. Severe bleeding was the only ECMO-related complication associated with a decreased ECMO survival (OR 0.48; 0.27-0.83 95\% CI) (Supplementary Information Fig. 5). Platelet count (OR 0.99; 0.995-0.999 95\% CI) and recent cancer therapy (OR 2.08; 1.1-3.92 95\% CI) were identified as independent risk factors for severe bleeding (Supplementary Information Fig. 6).

To assess whether the use of vv-ECMO in cancer patients with respiratory failure leads to outcomes that are superior to invasive mechanical ventilation alone, we compared the survival of patients from our cohort with propensity score-matched mechanically ventilated patients enrolled in the EFRAIM study [24]. 79 patients could be successfully matched to patients from the EFRAIM study. Results of propensity score-matching quality are shown in Supplementary Information Table 1. The standardized mean differences for each variable were substantially reduced after matching. After propensity score matching, there was no significant difference in the survival of patients receiving ECMO and those managed with mechanical ventilation only $(p=0.089)$ (Fig. 2). However, in patients with platelets above $250,000 / \mu \mathrm{l}$, the survival of patients receiving vv-ECMO was significantly higher than in patients receiving mechanical ventilation only ( $p=0.023$ ) (Supplementary Information Fig. 7).

To derive distinct patient subgroups with different trajectories within our study population, we performed LCA, a statistical method to uncover hidden subgroups in data. LCA can be used to classify patients into mutually exclusive and exhaustive subgroups of individuals, so called latent classes, based on their pattern of a set of categorical characteristics. The variables used for LCA were cancer type, age, $\mathrm{P} / \mathrm{F}$ ratio, platelets, remission 
status, cancer therapy in the previous 30 days, and duration of mechanical ventilation prior to vv-ECMO initiation. A 2-class LCA model best described the overall patient cohort (Fig. 3a). The two classes were practically identical to the subgroups of patients with hematologic malignancies and solid tumors, confirming the clinical significance of differentiating these patient populations. Moreover, there was a statistically significant difference in survival between these two groups (Fig. 3d, Class 1 vs. Class $1 p=0.0002$ ). We, therefore, performed LCA separately in patients with hematologic malignancies and solid tumors (Fig. 3b, c). In both groups, a 3-class model provided the best fit to the data. The three classes divided patients with hematologic malignancies and solid tumors into similar clinical subgroups. Class 1 consisted predominantly of older patients in partial remission who recently received tumor therapy but were not severely thrombocytopenic. The patients in class 2 were mostly younger and had all recently received tumor-directed therapy. As would be expected for younger patients treated with more aggressive regimens this patient population was characterized by moderate to severe thrombocytopenia. Class 3 represented a group of predominantly older patients who were either newly diagnosed with cancer or were in complete remission and therefore mostly not receiving any form of cancer therapy. Accordingly, these patients had no to mild thrombocytopenia. Importantly, the younger thrombocytopenic patients with hematologic malignancies undergoing active cancer therapy with severe thrombocytopenia, i.e., class 2 , had a worse survival than the patients in the other subgroups (Fig. 3e, Class 2 vs. Class $1 p=0.02$, Class 2 vs. Class $3 p=0.02$ ). None of the patients with solid tumors belonging to class 2 survived for more than 2 months after initiation of vv-ECMO (Fig. 3f, Class 2 versus Class $1 p=0.16$, Class 2 vs. Class $3 p=0.06$ ). However, the survival difference between class 1 and the other classes was not statistically significant.

\section{Discussion}

The main findings of our analysis show that disease status, low platelet count and high lactate levels indicate a poor prognosis. The outcome of cancer patients receiving ECMO was not superior to propensity score-matched patients treated only with conventional mechanical ventilation.

$\mathrm{Vv}$-ECMO represents a rescue intervention for patients with severe ARDS [25]. However, substantial uncertainty remains about the benefit of vv-ECMO for specific subgroups of patients [26, 27]. In particular, the role of vvECMO in cancer patients is a matter of debate. Only few studies have reported on outcomes of vv-ECMO in patients with malignant diseases [19, 28-34].

To our knowledge, the study presented here is the largest study in this patient population conducted to date. The only other larger study on this topic reported the outcome of 203 immunocompromised patients with severe

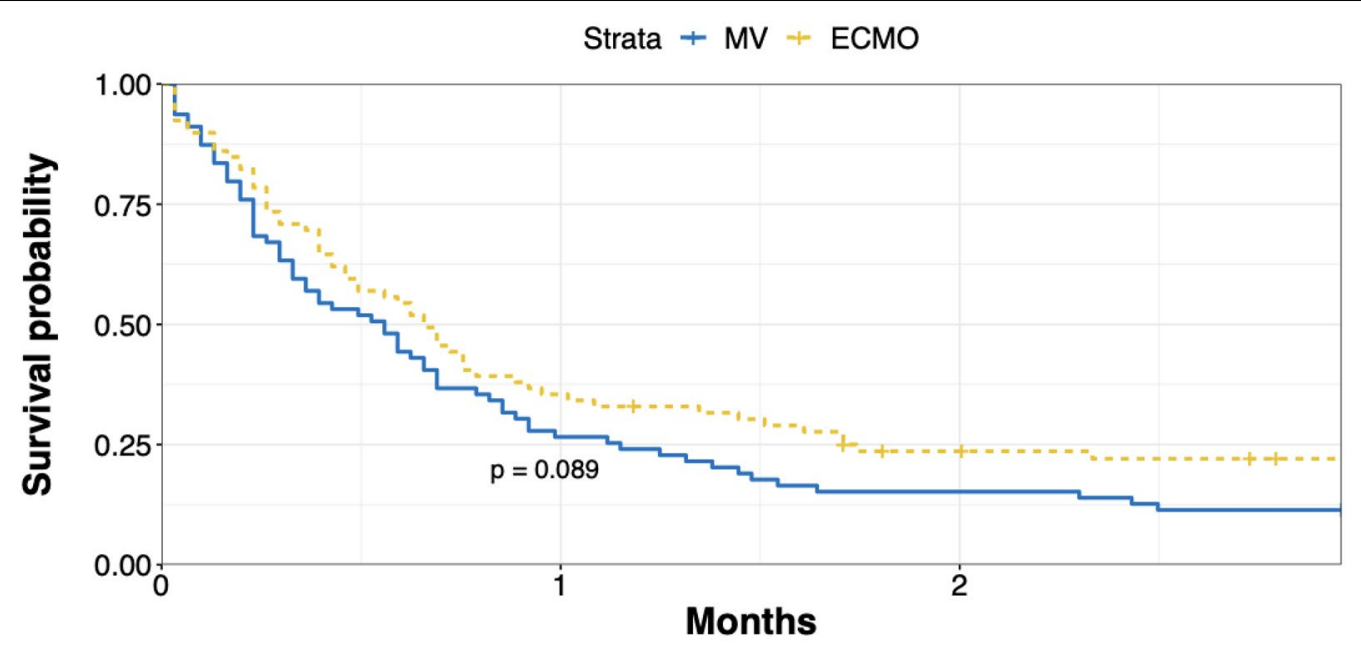

Number at risk

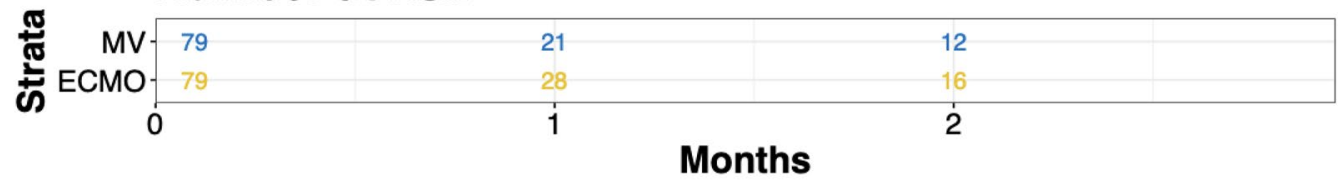

Fig. 2 Propensity score-matching patients receiving ECMO and those managed with mechanical ventilation (MV) only of the EFRAIM study [24] 
ARDS on vv-ECMO including 101 patients with cancer [19]. The study by Schmidt et al. investigated immunosuppressed patients, including patients with hematologic malignancies $(n=62)$, solid tumors $(n=39)$, solid-organ transplantation $(n=27)$, AIDS $(n=19)$, and long-term use of corticosteroids/immunosuppressants $(n=56)$. Overall, the outcome of cancer patients receiving vvECMO as a rescue treatment for respiratory failure was rather poor. The overall survival of cancer patients in this study was comparable to our study (6-month overall survival of $30 \%$ vs. a 60 -day overall survival of $26.8 \%$ in our study). Although the overall survival of cancer patients in our study was worse compared to patients without cancer, a significant minority of patients achieved long-term survival after vv-ECMO. Most deaths occurred in the first month after ECMO initiation. After around 60 days, the Kaplan-Meier overall survival curve reached a stable plateau (Supplementary information Fig. 1 and Fig. 2). The prognosis of patients with cancer on vv-ECMO should, therefore, not generally be regarded as futile. However, the high short-term mortality in our study indicates the need for better prognostic tools.

Even though the results are difficult to compare directly since Schmidt et al. only report the results for the overall population of immunosuppressed patients, there are important commonalities but also differences between the two studies. Identical to our study, platelet count was associated with overall survival in the study population of immunocompromised patients. Platelet count is a complex variable associated with and influenced by many clinical conditions. Potential causes for a low platelet count include myelosuppressive chemotherapy, bleeding, severe infection or sepsis, and severity of illness in many non-malignant medical conditions. Thus, in patients with cancer, platelet count can be regarded as an indicator of the general severity of illness and a risk factor for bleeding-related morbidity and mortality. Interestingly, LCA revealed that the severely thrombocytopenic patients were mostly younger cancer patients undergoing anticancer therapy. Unfortunately, even though such patients are usually aggressively treated with curative intent they seem to have a high vv-ECMO-associated mortality risk.
In addition, in the study by Schmidt et al. longer-term immunosuppression ( $>30 \mathrm{~d}$ ), baseline $\mathrm{PaCO} 2$, and driving pressure were shown to negatively affect outcome. In contrast, in our study, the group of patients with newly diagnosed cancer, i.e., cancer patients who are immunocompromised for only a short time, had a worse prognosis.

Treatment with vv-ECMO is associated with several serious and potentially life-threatening complications. The most frequent adverse events are bleeding, mechanical problems with the ECMO circuit, and device-related infections. Less common, but also severe complications include cerebrovascular events, cardiac arrhythmias, and cardiac arrest. The complication rates observed in the present analysis differ substantially from those reported in non-cancer patients (Table 2 and Supplementary Information Fig. 5) [16, 26]. We observed a high rate of severe bleeding episodes (38\%). Severe hemorrhage during ECMO was associated with increased ECMO-related mortality (Supplementary Information Fig. 5). Clinical factors that were correlated with an increased risk of bleeding were thrombocytopenia and recent chemotherapy (Supplementary Information Fig. 6). Thus, part of the negative prognostic impact of thrombocytopenia could be explained by higher risk of bleeding-related mortality during vv-ECMO, especially in combination with chemotherapy-induced vascular damage and disruption of the integrity of the skin and mucous barriers.

The provocative finding that the outcome of cancer patients receiving ECMO was not superior to propensity score-matched patients treated only with conventional mechanical ventilation, suggests that we currently have insufficient knowledge to appropriately select patients who would benefit from provision of vv-ECMO support. To improve the outcome of cancer patients with acute respiratory failure, it will be crucial to better understand which clinical characteristics are important for patient selection and how these factors affect the outcome of vv-ECMO. In this study, the presence of thrombocytopenia was consistently associated with worse outcome and should be accounted for in the decision to offer vvECMO support. Since increased bleeding-related complications seem to be an important contributing factor

\footnotetext{
(See figure on next page.)

Fig. 3 Latent class analysis and corresponding Kaplan-Meier curves for survival probability for all patients (a, d), patients with hematologic malignancies. (b, e), and patients with solid tumors (c, f). The overall patient cohort is best described by a 2-class model, which divides the cohort into patients with hematologic malignancies and solid tumors (d. Class 1 vs. Class $1 p=0.0002$ ) Patients with hematologic malignancies (b) are divided into three analogous classes. Class 1: patients who are more likely to be older and in partial remission with only mild thrombocytopenia. Class 2 : younger patients undergoing cancer therapy with moderate to severe thrombocytopenia. Class 3: predominantly elderly patients who currently do not undergo cancer treatment because they are newly diagnosed or in complete remission and have no to mild thrombocytopenia. Class 2 had a worse survival than the patients in the other subgroups (i.e., Class 2 vs. Class $1 p=0.02$, Class 2 vs. Class $3 p=0.02$ ). All patients with solid tumors belonging to class 2 died within one month after initiation of vv-ECMO (c). (Abbreviations: complete remission (CR); partial remission (PR); progressive disease (PD)). Related gradiations have been color-coded in the categories
} 
a

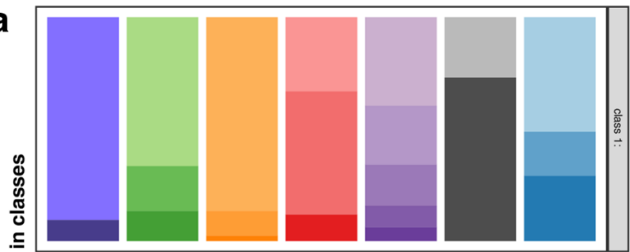

든

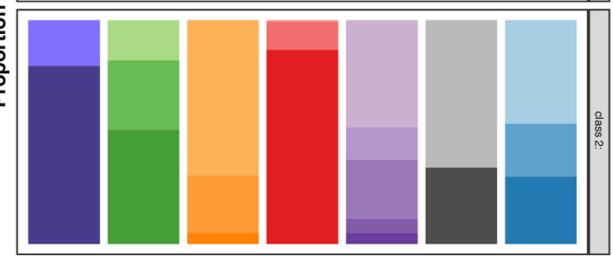

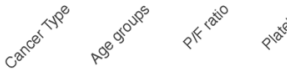

b

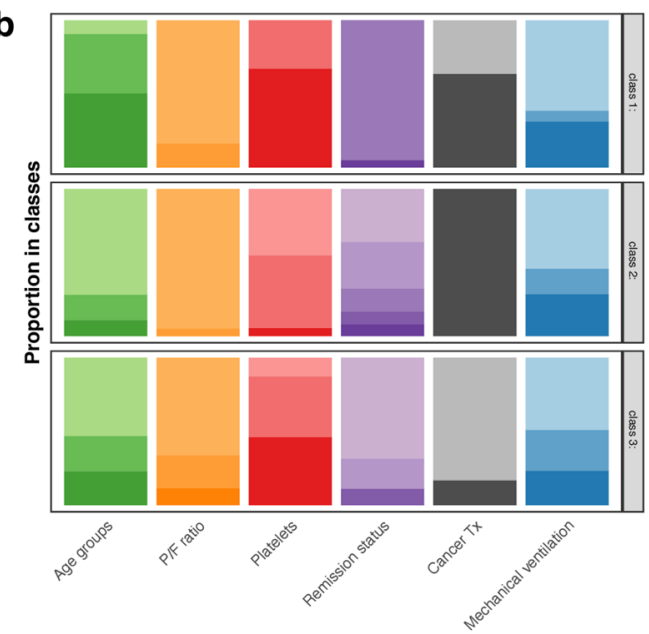

C

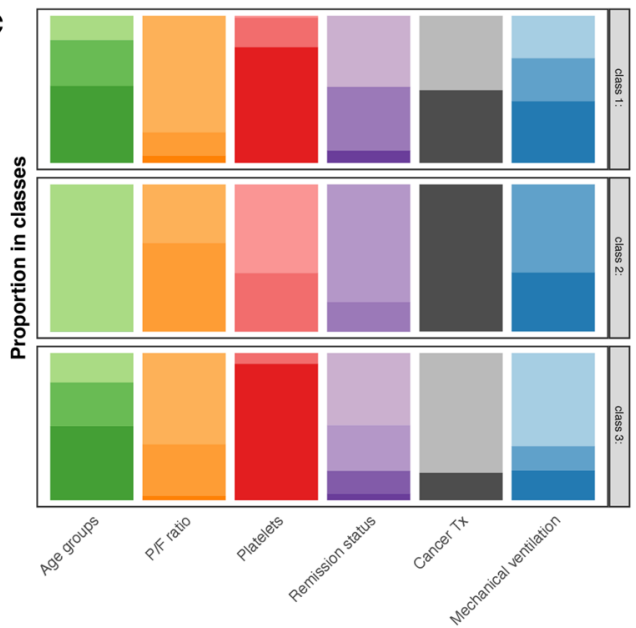

d
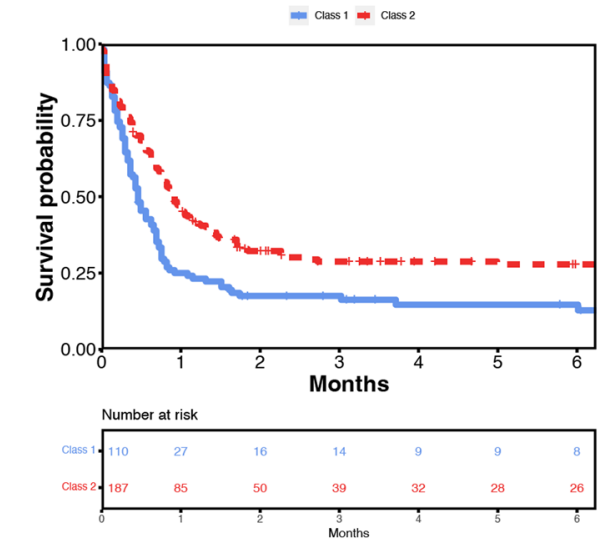

e
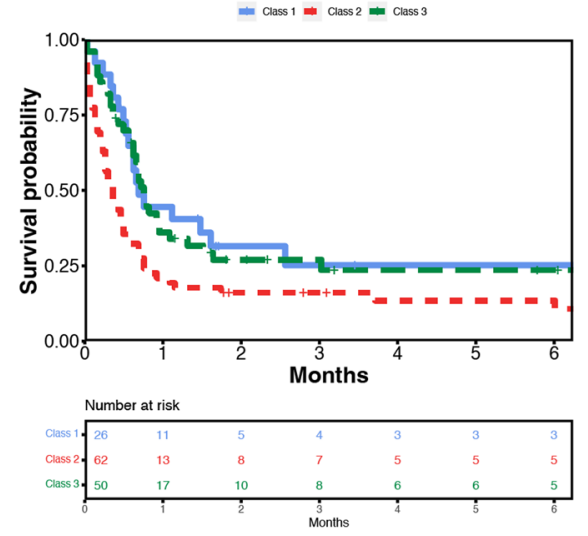

f
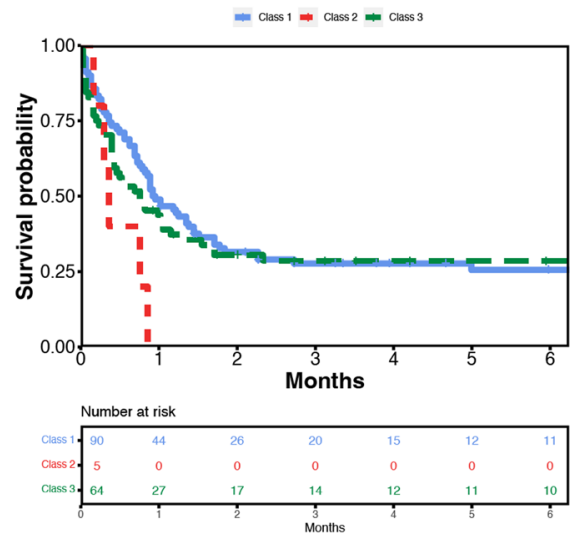

Cancer type Age groups P/F ratio Platelets

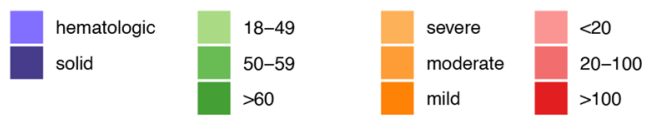

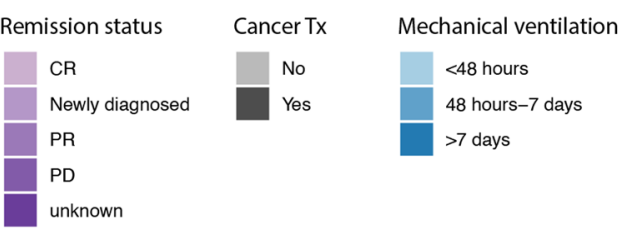


for poor outcome in thrombocytopenic patients, further improvements in coagulation and bleeding management are needed and should lead to higher rates of treatment success with vv-ECMO in cancer patients.

Furthermore, this study demonstrates that a relevant number of patients $(8 \%)$ received vv-ECMO despite progression of their malignant disease at the time of ECMO initiation. The decision to initiate vv-ECMO in this setting is intriguing given that tumor progression is generally associated with a poor prognosis [36-38]. This study confirms that progressive disease indicates a very poor prognosis (Fig. 1). Only $3 / 22$ (13\%) patients with progressive disease survived. Patients with progressive disease, therefore, should not be offered vv-ECMO.

The present study has several limitations. Due to the retrospective study design, selection bias and residual confounding cannot be excluded. We included a wide variety of variables to adjust for differences in patient characteristics, but further variance remains which we were not able to account for. The hospitals included in the study were not a random sample of all hospitals performing ECMO in cancer patients. The majority were large tertiary care hospitals with experience in vv-ECMO as well as in providing care to critically ill patients with cancer and other complex conditions such as severe respiratory failure. In addition, the collected data span over a period of 10 years. Over the last decade, the management of patients with ARDS, including those undergoing vv-ECMO has improved considerably. Concurrently, there have been advances in the treatment of cancer during the study period. We therefore cannot exclude time-dependent effects that may have influenced our analysis. However, a comparison between the time periods 2009-2013 and 2014-2019 showed no major differences (Supplementary information Table 3). In the matched-pair analysis of patients treated with ECMO or mechanical ventilation only, we tried to match patients for relevant clinical characteristics. Nonetheless, despite propensity score matching, there may be additional unaccounted confounders that could affect the results of our analysis. The results of the LCA should be interpreted with caution due to the limited sample size, particularly for the subpopulations of hematologic malignancies and solid tumor.

\section{Conclusion}

The overall survival of cancer patients who require vv-ECMO is poor. This study shows that the value of

Table 2 Major vv-ECMO-related complications or complications during vv-ECMO

\begin{tabular}{|c|c|c|c|c|}
\hline & Overall & $\begin{array}{l}\text { Hematologic malignan- } \\
\text { cies }\end{array}$ & Solid tumors & $p$-value \\
\hline No. of patients (\%) & 297 & $138(46.4)$ & $159(53.5)$ & $<0.001$ \\
\hline Packed red blood cells units (\%) & & & & 0.974 \\
\hline 0 & $16(5.4)$ & $8(5.8)$ & $8(5)$ & \\
\hline $1-5$ & $69(23.2)$ & $31(22.5)$ & $38(23.9)$ & \\
\hline $5-10$ & $56(18.9)$ & $27(19.6)$ & $29(18.2)$ & \\
\hline$>10$ & $156(52.5)$ & $72(52.2)$ & $84(52.8)$ & \\
\hline Platelet units (\%) & & & & $<0.001$ \\
\hline 0 & $110(37)$ & $28(20.3)$ & $82(51.6)$ & \\
\hline $1-5$ & $81(27.3)$ & $40(29)$ & $41(25.8)$ & \\
\hline $5-10$ & $37(12.5)$ & $16(11.6)$ & $21(13.2)$ & \\
\hline$>10$ & $69(23.2)$ & $54(39.1)$ & $15(9.4)$ & \\
\hline Severe bleeding (\%) & $113(38)$ & $61(44.2)$ & $52(32.7)$ & 0.055 \\
\hline Ischemic stroke (\%) & $11(3.7)$ & $4(2.9)$ & $7(4.4)$ & 0.707 \\
\hline vv-ECMO system changed (\%) & $46(15.5)$ & $15(10.9)$ & $31(19.5)$ & 0.059 \\
\hline Number of vv-ECMO system changes (\%) & & & & 0.109 \\
\hline 0 & $251(84.5)$ & $123(89.1)$ & $128(80.5)$ & \\
\hline 1 & $33(11.1)$ & $9(6.5)$ & $24(15.1)$ & \\
\hline 2 & $11(3.7)$ & $5(3.6)$ & $6(3.8)$ & \\
\hline 3 & $1(0.3)$ & $1(0.7)$ & $0(0)$ & \\
\hline 5 & $1(0.3)$ & $0(0)$ & $1(0.6)$ & \\
\hline Accidental decannulation (\%) & 0 & $0(0)$ & $0(0)$ & NA \\
\hline Cardiac arrest during vv-ECMO (\%) & $30(10.1)$ & $18(13)$ & $12(7.5)$ & 0.169 \\
\hline Ventilator associated pneumothorax (\%) & $33(11.1)$ & $13(9.4)$ & $20(12.6)$ & 0.497 \\
\hline
\end{tabular}


$\mathrm{vv}$-ECMO in cancer patients with respiratory failure is still unclear and further research is needed. Given the uncertainty regarding the value of vv-ECMO in this patient population the indication for vv-ECMO should be made by a multidisciplinary team including both intensivists and hemato-oncologists taking into account both the prognosis of the acute condition leading to ICU admission and the underlying malignancy. In this study, we identified disease status, low platelet count and high lactate levels as indicators of poor prognosis that should be accounted for in the decision to provide vv-ECMO.

\section{Supplementary Information}

The online version contains supplementary material available at https://doi. org/10.1007/s00134-022-06635-y.

\begin{abstract}
Author details
${ }^{1}$ First Department of Internal Medicine, Faculty of Medicine and University Hospital Cologne, Center of Integrated Oncology Aachen Bonn Cologne Dusseldorf, University of Cologne, University Hospital Cologne, Kerpener Str. 62, 50937 Cologne, Germany. ${ }^{2}$ Department of Hematology, Hemostasis, Oncology and Stem Cell Transplantation, Medical School, Hannover, Germany. ${ }^{3}$ Department of Anesthesiology, University UIm, Ulm, Germany. ${ }^{4}$ Department of Intensive Care Medicine, University Medical Center Hamburg-Eppendorf, Hamburg, Germany. ${ }^{5}$ Medical University of Graz, Graz, Austria. ${ }^{6}$ Department of Internal Medicine B, Medical Intensive Care Unit, University Medicine Greifswald, Greifswald, Germany. ${ }^{7}$ Klinikum Oldenburg, Oldenburg, Germany. ${ }^{8}$ Department Medicine II, Intensive Care Unit 13H3, Medical University Vienna, Vienna, Austria. ${ }^{9}$ Städtische Kliniken Köln Merheim, University Witten/Herdecke, Herdecke, Germany. ${ }^{10}$ Klinikum Emden, Emden, Germany. ${ }^{11}$ Evangelisches Krankenhaus Oldenburg, Oldenburg, Germany. ${ }^{12}$ Department of Internal Medicine $\mathrm{V}$ - Pneumology, Allergology and Intensive Care Medicine, University Hospital of Saarland, Homburg, Germany. ${ }^{13}$ Department of Hematology and Stem Cell Transplantation, University Hospital Essen, University of Duisburg-Essen, Essen, Germany. ${ }^{14}$ University, Würzburg, Germany.

${ }^{15}$ Robert-Bosch-Krankenhaus Gerlingen and Stuttgart, Stuttgart, Germany.

${ }^{16}$ Department of Anesthesiology and Intensive Care Medicine, University Hospital Bonn, Bonn, Germany. ${ }^{17}$ Department Medicine I, Intensive Care Unit 13i2, Medical University Vienna, Vienna, Austria. ${ }^{18}$ Klinikverbund Allgäu, Klinik Immenstadt, Immenstadt, Germany. ${ }^{19}$ University Hospital Dresden, Dresden, Germany. ${ }^{20}$ Department of Anesthesiology and Operative Intensive Care Medicine Charite, Berlin, Germany. ${ }^{21}$ Center for Acute Respiratory Failure, New York-Presbyterian Medical Center, New York, NY, USA. ${ }^{22}$ Division of Pulmonary, Allergy and Critical Care Medicine, Department of Medicine, Columbia University College of Physicians and Surgeons, New York, NY, USA. ${ }^{23}$ Médecine Intensive Et Réanimation, APHP, Saint-Louis Hospital and Paris University, Paris, France.
\end{abstract}

\section{Acknowledgements}

The following authors are members of the ARDS Netzwerk: MK, SK, SF, PS, $J C S, S B, C K, P L, R M, C P, S W C$. The following authors are members of the iCHOP working group: MK, ASV, DAE, BB, TL, CL, GB, PS. We thank all members and associated centers of the iCHOP working group for enabling the conduct of this retrospective study which provided a basis for this analysis: Jorge Garcia Borrega, Thomas Staudinger, Wolfgang R. Sperr, Paul Knöbl, Philipp Wohlfarth and Alexander Carlström. We thank all contributors to the EFRAIM study, especially Michael Darmon for helpful advice and discussion. We thank also the Nine-l investigators and the members of the ARDS Netzwerk.

\section{Author contributions}

Conception and design: MK, JK, BB, DAE, AS-V. Provision of study materials or patients: All authors. Collection and assembly of data: All authors. Data analysis and interpretation: MK, JK, BB, DAE, AS-V, MH, CK, CP, DB, EA. Manuscript writing: MK, JK, BB, DAE, AS-V. Final approval of manuscript: All authors.
Funding

Open Access funding enabled and organized by Projekt DEAL.

\section{Declarations}

\section{Conflicts of interest}

PS has received speaker fees by Getinge. CK is consultant for Xenios AG. Remaining authors declare that they have no conflict of interest. This research did not receive any specific grant from funding agencies in the public, commercial or non-profit sectors.

\section{Open Access}

This article is licensed under a Creative Commons Attribution-NonCommercial 4.0 International License, which permits any non-commercial use, sharing, adaptation, distribution and reproduction in any medium or format, as long as you give appropriate credit to the original author(s) and the source, provide a link to the Creative Commons licence, and indicate if changes were made. The images or other third party material in this article are included in the article's Creative Commons licence, unless indicated otherwise in a credit line to the material. If material is not included in the article's Creative Commons licence and your intended use is not permitted by statutory regulation or exceeds the permitted use, you will need to obtain permission directly from the copyright holder. To view a copy of this licence, visit http://creativecommons.org/licen ses/by-nc/4.0/.

\section{Publisher's Note}

Springer Nature remains neutral with regard to jurisdictional claims in published maps and institutional affiliations.

Received: 29 September 2021 Accepted: 24 January 2022

Published online: 10 February 2022

\section{References}

1. Pastores $S M$, Voigt LP (2010) Acute respiratory failure in the patient with cancer: diagnostic and management strategies. Crit Care Clin 26:21-40. https://doi.org/10.1016/j.ccc.2009.10.001

2. Azoulay E, Lemiale V, Mourvillier B et al (2018) Management and outcomes of acute respiratory distress syndrome patients with and without comorbid conditions. Intens Care Med 44:1050-1060. https://doi.org/10. 1007/s00134-018-5209-6

3. Neuschwander A, Lemiale V, Darmon M et al (2017) Noninvasive ventilation during acute respiratory distress syndrome in patients with cancer: Trends in use and outcome. J Crit Care 38:295-299. https://doi.org/10. 1016/j.jcrc.2016.11.042

4. Kochanek M, Shimabukuro-Vornhagen A, Russ K et al (2020) Prevalence of cancer patients in German intensive care units. Medizinische Klinik - Intensivmedizin Und Notfallmedizin 115:312-319. https://doi.org/10. 1007/s00063-019-0594-3

5. Kiehl MG, Beutel G, Boll B et al (2018) Consensus statement for cancer patients requiring intensive care support. Ann Hematol 97:1271-1282. https://doi.org/10.1007/s00277-018-3312-y

6. Azoulay E, Mokart D, Pene F et al (2013) Outcomes of critically ill patients with hematologic malignancies: prospective multicenter data from France and Belgium-a groupe de recherche respiratoire en reanimation onco-hematologique study. J Clin Oncol 31:2810-2818. https://doi.org/ 10.1200/jco.2012.47.2365

7. Force ADT, Ranieri VM, Rubenfeld GD et al (2012) Acute respiratory distress syndrome: the Berlin definition. JAMA 307:2526-2533. https://doi. org/10.1001/jama.2012.5669

8. Fan E, Brodie D, Slutsky AS (2018) Acute respiratory distress syndrome: advances in diagnosis and treatment. JAMA 319:698-710. https://doi.org/ 10.1001/jama.2017.21907

9. Dumas G, Lemiale V, Rathi N et al (2021) Survival in immunocompromised patients ultimately requiring invasive mechanical ventilation: a pooled individual patient data analysis. Am J Resp Crit Care. https://doi. org/10.1164/rccm.202009-3575oc 
10. Bellani G, Laffey JG, Pham T et al (2016) Epidemiology, patterns of care, and mortality for patients with acute respiratory distress syndrome in intensive care units in 50 countries. JAMA 315:788-800. https://doi.org/ 10.1001/jama.2016.0291

11. Sud S, Friedrich JO, Taccone P et al (2010) Prone ventilation reduces mortality in patients with acute respiratory failure and severe hypoxemia: systematic review and meta-analysis. Intens Care Med 36:585-599. https://doi.org/10.1007/s00134-009-1748-1

12. Mercat A, Richard JC, Vielle B et al (2008) Positive end-expiratory pressure setting in adults with acute lung injury and acute respiratory distress syndrome: a randomized controlled trial. JAMA 299:646-655. https://doi. org/10.1001/jama.299.6.646

13. Baek MS, Lee SM, Chung CR et al (2019) Improvement in the survival rates of extracorporeal membrane oxygenation-supported respiratory failure patients: a multicenter retrospective study in Korean patients. Crit Care 23:1. https://doi.org/10.1186/s13054-018-2293-5

14. Karagiannidis C, Brodie D, Strassmann S et al (2016) Extracorporeal membrane oxygenation: evolving epidemiology and mortality. Intens Care Med 42:889-896. https://doi.org/10.1007/s00134-016-4273-z

15. Combes A, Schmidt M, Hodgson CL et al (2020) Extracorporeal life support for adults with acute respiratory distress syndrome. Intens Care Med 46:2464-2476. https://doi.org/10.1007/s00134-020-06290-1

16. Thiagarajan RR, Barbaro RP, Rycus PT et al (2017) Extracorporeal life support organization registry international report 2016. Asaio J 63:60-67. https://doi.org/10.1097/mat.0000000000000475

17. Schmidt M, Brechot N, Combes A (2016) Ten situations in which ECMO is unlikely to be successful. Intens Care Med 42:750-752. https://doi.org/10. 1007/s00134-015-4013-9

18. Schmidt M, Combes A, Shekar K (2019) ECMO for immunosuppressed patients with acute respiratory distress syndrome: drawing a line in the sand. Intens Care Med 45:1140-1142. https://doi.org/10.1007/ s00134-019-05632-y

19. Schmidt $M$, Schellongowski P, Patroniti N et al (2018) Six-month outcome of immunocompromised patients with severe acute respiratory distress syndrome rescued by extracorporeal membrane oxygenation. an international multicenter retrospective study. Am J Respir Crit Care Med 197:1297-1307. https://doi.org/10.1164/rccm.201708-1761 oc

20. Biffi S, Bella SD, Scaravilli V et al (2017) Infections during extracorporeal membrane oxygenation: epidemiology, risk factors, pathogenesis and prevention. Int J Antimicrob Ag 50:9-16. https://doi.org/10.1016/j.ijant imicag.2017.02.025

21. Shimabukuro-Vornhagen A, Boll B, Kochanek M et al (2016) Critical care of patients with cancer. Ca Cancer J Clin 66:496-517. https://doi.org/10. 3322/caac. 21351

22. Tonna JE, Abrams D, Brodie D et al (2021) Management of adult patients supported with venovenous extracorporeal membrane oxygenation (V ECMO): guideline from the extracorporeal life support organization (ELSO). Asaio J 67:601-610. https://doi.org/10.1097/mat.0000000000 001432

23. Schulman S, Kearon C, the SS on C of A of, et al (2005) Definition of major bleeding in clinical investigations of antihemostatic medicinal products in non-surgical patients. J Thromb Haemost 3:692-694. https://doi.org/ 10.1111/j.1538-7836.2005.01204.x

24. Azoulay E, Pickkers P, Soares M et al (2017) Acute hypoxemic respiratory failure in immunocompromised patients: the Efraim multinational prospective cohort study. Intens Care Med 43:1808-1819. https://doi.org/ 10.1007/s00134-017-4947-1
25. Mi MY, Matthay MA, Morris AH (2018) Extracorporeal membrane oxygenation for severe acute respiratory distress syndrome. New Engl J Med 379:884-887. https://doi.org/10.1056/nejmclde1804601

26. Combes A, Hajage D, Capellier G et al (2018) Extracorporeal membrane oxygenation for severe acute respiratory distress syndrome. New Engl J Med 378:1965-1975. https://doi.org/10.1056/nejmoa1800385

27. Munshi L, Telesnicki T, Walkey A, Fan E (2014) Extracorporeal life support for acute respiratory failure. A systematic review and metaanalysis. Ann Am Thorac Soc 11:802-810. https://doi.org/10.1513/annalsats. 201401-0120c

28. Schmidt M, Brodie D, Combes A (2014) Patients with hematologic malignancies have many reasons to die during extracorporeal membrane oxygenation. Crit Care 18:522. https://doi.org/10.1186/s13054-014-0522-0

29. Wohlfarth P, Ullrich R, Staudinger T et al (2014) Extracorporeal membrane oxygenation in adult patients with hematologic malignancies and severe acute respiratory failure. Crit Care 18:R20. https://doi.org/10.1186/cc137 01

30. Gow KW, Heiss KF, Wulkan ML et al (2009) Extracorporeal life support for support of children with malignancy and respiratory or cardiac failure: The extracorporeal life support experience. Crit Care Med 37:1308-1316. https://doi.org/10.1097/ccm.0b013e31819cf01a

31. Park PK (2017) Extracorporeal membrane oxygenation support following stem cell transplant-when is all that we have still not enough? Crit Care Med 45:925-926. https://doi.org/10.1097/ccm.0000000000002387

32. Wohlfarth P, Beutel G, Lebiedz P et al (2017) Characteristics and outcome of patients after allogeneic hematopoietic stem cell transplantation treated with extracorporeal membrane oxygenation for acute respiratory distress syndrome. Crit Care Med 45:e500-e507. https://doi.org/10.1097/ ccm.0000000000002293

33. Kang HS, Rhee CK, Lee HY et al (2015) Clinical outcomes of extracorporeal membrane oxygenation support in patients with hematologic malignancies. Korean J Intern Medicine 30:478-488. https://doi.org/10.3904/kjim. 2015.30.4.478

34. Wolfson RK, Kahana MD, Nachman JB, Lantos J (2005) Extracorporeal membrane oxygenation after stem cell transplant: clinical decision-making in the absence of evidence. Pediatr Crit Care Me 6:200-203. https:// doi.org/10.1097/01.pcc.0000155635.02240.9c

35. Lazzeri C, Bonizzoli M, Cianchi G et al (2018) Lactate and echocardiography before veno-venous extracorporeal membrane oxygenation support. Hear Lung Circulation 27:99-103. https://doi.org/10.1016/j.hlc.2017.02. 006

36. Hilder M, Herbstreit F, Adamzik M et al (2017) Comparison of mortality prediction models in acute respiratory distress syndrome undergoing extracorporeal membrane oxygenation and development of a novel prediction score: the PREdiction of Survival on ECMO Therapy-Score (PRESETScore). Crit Care 21:301. https://doi.org/10.1186/s13054-017-1888-6

37. Piccinni P, Cruz DN, Gramaticopolo S et al (2011) Prospective multicenter study on epidemiology of acute kidney injury in the ICU: a critical care nephrology Italian collaborative effort (NEFROINT). Minerva Anestesiol 77:1072-1083

38. Seylanova N, Crichton S, Zhang J et al (2020) Acute kidney injury in critically ill cancer patients is associated with mortality: A retrospective analysis. PLoS ONE 15:e0232370. https://doi.org/10.1371/journal.pone. 0232370 